\title{
Automation for clinicians in the field: The validity of a performance support system
}

\author{
MATTHEW G. HILE \\ University of Missouri-Columbia at the Missouri Institute of Mental Health, St. Louis, Missouri \\ DONNA M. CAMPBELL \\ Webster University, St. Louis, Missouri \\ and \\ BAGHER GHOBARY \\ University of Missouri-Columbia at the Missouri Institute of Mental Health, St. Louis, Missouri
}

\begin{abstract}
The Mental Retardation-Expert (MR-E) is an electronic performance support system for clinicians who work with mentally retarded persons who have behavioral disorders. It provides treatment planning assistance when needed. This study compares MR-E's advice with that offered by human experts. It assesses the impact of the system on clinicians from various areas. The results support MR-E's validity and its ability to help clinicians function as experts. They highlight potential benefits for psychologists in using performance support systems.
\end{abstract}

Effective clinical treatment depends on clinicians' access to current scientific knowledge and expertise, as well as on the ability to apply that information to individual cases. Although professional education prepares individuals for this process, clinical experience and continuing education transform novices into effective practitioners. Traditionally, practitioners have relied on scientific publications, supervision, and continuing education to maintain and enhance their skills. A new tool, the performance support system, may offer a more effective and efficient alternative.

Performance support systems (PSSs) are computer programs designed "to provide whatever is necessary to generate performance and learning at the moment of need" (Gery, 1991, p. 34). Such programs use "knowledgebased systems, hypertext, on-line reference, extensive data bases, and allied technologies to provide support to performers on the job, where they need it, when they need it, in the form most useful to them" (Carr, 1991, p. 46). PSSs fill a variety of support roles. They function as librarians (collecting, organizing, and presenting information needed to perform a job), as advisors (providing the necessary expert assistance), or as tutors (teaching individuals how to perform specific tasks).

The Mental Retardation-Expert project is funded in part by the $\mathrm{Na}$ tional Institute of Mental Health (Grant 1 R29 MH43439). The opinions expressed in this paper are those of the authors, and no official endorsement by any agency should be inferred. MR-E will not be available for clinical use until its reliability and validity have been well established. The authors gratefully acknowledge the assistance of Stephanie Patag and the editorial assistance of Linda Sage. Correspondence should be sent to M. G. Hile, Missouri Institute of Mental Health, 5247 Fyler Ave., St. Louis, MO 63139-1494 (e-mail: medmip@mizzoul.missouri.edu).
The Mental Retardation-Expert (MR-E) is a PSS for psychologists who treat severe behavior problems that are exhibited by some persons with mental retardation. It combines current scientific literature, examples of behavioral treatment programs, a behavioral glossary, and an expert treatment planning component to help practitioners provide effective treatments (Hile \& Desrochers, in press). MR-E is designed to improve the expertise of inexperienced professionals and to provide expert consultation to clinicians when and where it is needed.

Modeled on a human expert (Hile, Campbell, Ghobary, \& Desrochers, 1993), MR-E's expert consultation facility asks clinicians a series of yes/no questions to help determine the function of a particular undesirable behavior. On the basis of the resulting functional hypotheses, MR-E identifies treatments specifically targeted toward reducing the undesirable behaviors, most often by replacing them with more desirable alternatives. For example, if aggression allows an individual to escape from a disliked activity, a functionally based treatment might teach the person to request a break. For the individual who obtains staff attention for aggression, a functional approach would teach socially appropriate ways to begin and maintain conversations with staff. Axelrod (1987) has suggested that functionally specific interventions may increase both treatment effectiveness and efficiency.

From its consultations, MR-E generates reports that (1) list the functional hypotheses used to develop treatment plans, (2) discuss how those functions affect client behaviors, and (3) describe recommended treatment procedures for each client. These reports serve as the basis of our evaluation in this study.

Because MR-E's expert consultation combines diagnostic assessment and treatment recommendations, its valid- 
ity must be established. Two points require consideration. First, how closely does MR-E's advice approach the expert advice that produced the system? To answer this, we determined the absolute level of agreement between MR-E and our human expert, as well as the level of agreement between MR-E and other domain experts. Second, does assistance from MR-E change the thoughts or behaviors of its users?

\section{METHOD}

We created two fictional case descriptions of the aggressive behaviors of "Jim," a 19-year-old man with moderate mental retardation who worked in a sheltered workshop. The descriptions differed only in terms of the function of aggression. In one instance, aggression allowed Jim to escape a disliked task; in the other, it got him additional staff attention.

We obtained experimental packets from three experts (including the expert on whom MR-E is based) and 18 clinicians with various levels of experience in treating persons with mental retardation. The experts were paid for their participation; the clinicians volunteered. The packets included a brief description of MR-E, a definition of functional hypotheses, a demographic questionnaire, and a set of pretest and posttest materials. In the pretest, participants received one of the two case descriptions, a free-form data sheet to record their functional hypotheses and treatment recommendations, a checklist to identify functional hypotheses, and a checklist to identify treatment selections. The posttest provided the MR-E consultation report for the case description along with the free-form data sheet and checklists included in the pretest. The instructions specifically mentioned that participants could review any pretest materials when completing these posttest questionnaires. Finally, each packet contained a questionnaire asking for the rater's evaluation of the MR-E consultation report. Each clinician rated a single, randomly selected, case description; the three experts evaluated both cases.

The experts, three men, were nationally recognized individuals with numerous publications, including books, and presentations. Their clinical experience ranged from 18 to 35 years and, in addition to providing clinical consultations, all held academic appointments. The clinicians, 13 female and 5 male, reported clinical experience of $0-15$ years $(M=6.78, S D=4.32)$. Eleven clinicians provided direct client services, six were administrators, and one was a university professor. Between them, they had a broad range of formal academic training: three held high-school diplomas, seven had bachelor's degrees, four had earned master's degrees, and four held doctoral degrees.

\section{RESULTS}

\section{MR-E Compared With Human Experts}

To compare MR-E's advice with that offered by human experts, we examined agreement on functional hypotheses between the human and automated experts. Functional hypotheses serve as the basis of all of MR-E's treatment suggestions. Agreement was compared by using interobserver reliability (IOR) scores, which range from 0 (no agreement) to 1 (complete unanimity) (Kazdin, 1982). Table 1 lists IOR scores for each pair of experts.

The absolute level of functional hypothesis agreement between MR-E and Expert A, upon whom the system is based, was quite high in the pretest $(\mathrm{IOR}=.86)$. Dis-
Table 1

Pre- and Posttest Interobserver Reliability Scores (IORs)

for Functional Hypotheses Between the Three Experts and Mental Retardation-Expert (MR-E)

\begin{tabular}{ccccc} 
& \multicolumn{4}{c}{ Consultation } \\
\cline { 2 - 4 } Consultation & Expert & MR-E \\
\cline { 2 - 4 } Exper & & B & C & \\
A & & .81 & .88 & 1.00 \\
B & .74 & & .79 & .81 \\
C & .79 & .76 & & .93 \\
MR-E & .86 & .79 & .93 & \\
\hline
\end{tabular}

Note-Posttest IORs are in the upper right quadrant.

agreements resulted from Expert A's selection of additional potential hypotheses not reported by MR-E. For both case descriptions, the most probable hypotheses selected by Expert A were also chosen by MR-E. Disagreements disappeared in the posttest, when Expert $A$ and MR-E provided identical answers (IOR $=1$ ).

We next compared the agreements between the three human experts with those between MR-E and those experts. The IORs between the three human experts ranged from .74 in the pretest comparison between Experts A and $\mathrm{B}$, to .88 , for the posttest comparison between Experts $A$ and $C$. The level of agreement between MR-E and Expert $\mathrm{A}$ is higher in the pretest and posttest than that between any other pair of human experts in those conditions. MR-E's level of agreement with each expert, ranging from .79 to 1 , compares favorably with the range of agreement between human experts.

\section{MR-E's Impact on Clinicians}

To address our second point, the impact of MR-E's advice on clinicians, we began by examining the level of agreement between the clinician users and the various experts (see Figure 1). Agreement between the clinicians and MR-E was again quite high, ranging from a pretest value of .88 to a posttest value of .94 . It was significantly higher on the posttest than on the pretest $[t(17)=3.72$, $p<.001]$.

In addition to agreement level, it is important to examine changes in both sensitivity and specificity of clinicians' judgments (Fischer, Parides, \& Davies, 1993) that result from an MR-E consultation report. Specificity refers to the precision with which clinicians can identify particular functional hypotheses. Sensitivity refers to the precision with which clinicians can correctly reject inappropriate functional hypotheses.

Between pretest and posttest, the clinicians' average specificity rose significantly from .92 to $.95[t(17)=$ $2.03, p<.05]$. This means that clinicians were able to correctly identify $95 \%$ of the existing functional hypotheses by using MR-E's consultation. Sensitivity also rose significantly from a pretest mean of .67 to a posttest mean of $.91[t(17)=3.71, p<.001]$. Before receiving an MR-E consultation, clinicians were correctly rejecting 


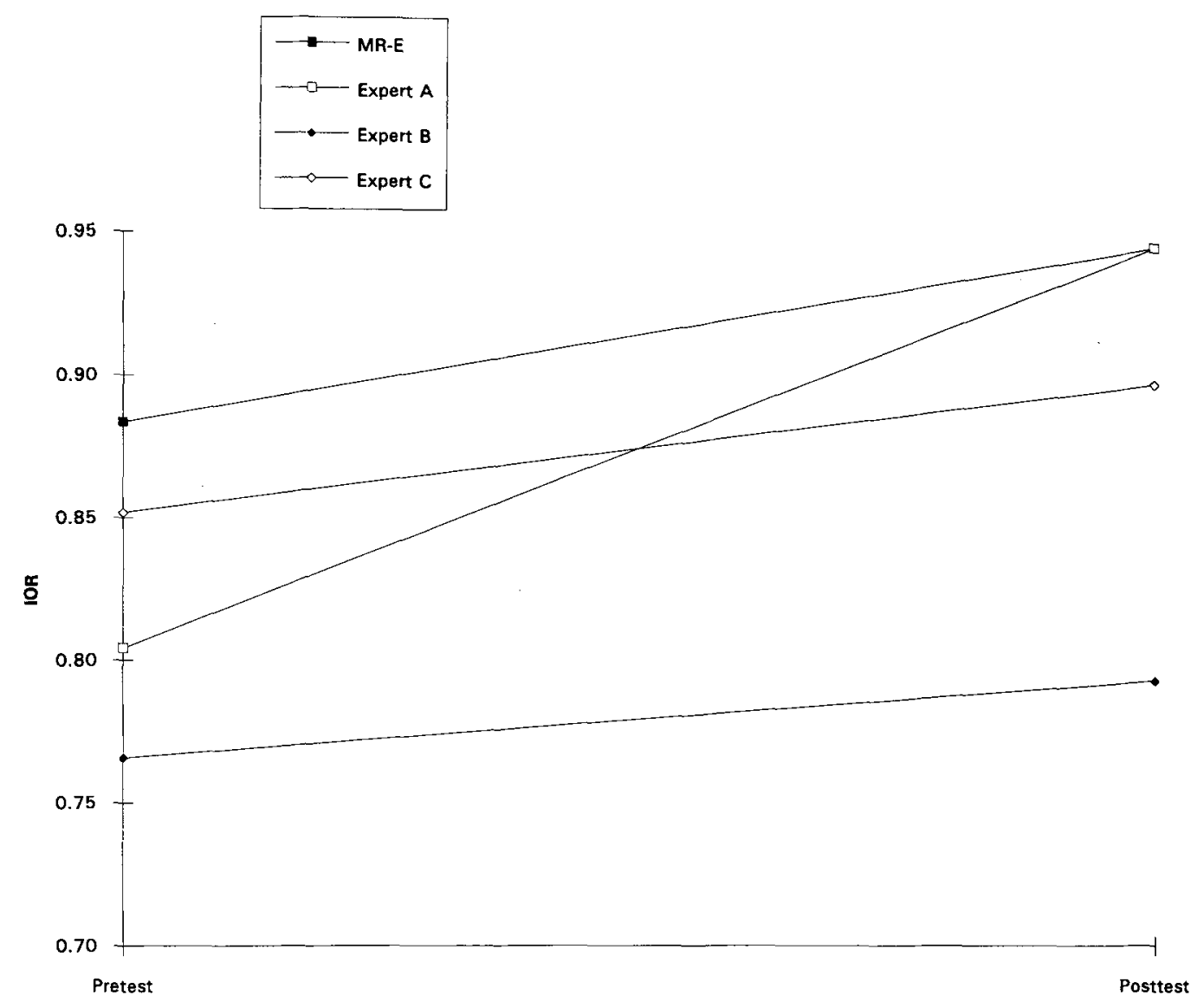

Figure 1. Agreement between clinicians and human/automated experts.

only $67 \%$ of the functional hypotheses. After a consultation, they correctly rejected $91 \%$ of these hypotheses.

We also examined the impact of MR-E's advice on treatment selections. We constructed our two cases to recommend the same four treatments, allowing us to compare the treatment choices of all participants. The pretest and posttest endorsements of these treatments by the clinicians were analyzed with both a paired $t$ test and the McNemar test. The latter is a nonparametric statistic for analyzing dichotomous variables (Norusis, 1992). For two of the recommended treatments-breaking the behavioral chain leading to aggression $[t(17)=2.92, p<.005$, McNemar $p<.02]$ and communication training $[t(17)=$ $3.29, p<.001$, McNemar $p<.01]$-both tests indicated a significant increase in posttest recommendations. The pattern of significant test results for the other twoincreasing positive reinforcement $[t(17)=1.84, p<.02$, McNemar $p$, n.s.] and developing specific skills to replace the aggressive behavior $[t(17)=2.20, p<.01, \mathrm{McNe}-$ mar $p<.06]$ - was less stable but still indicated an increase in recommended usage on posttest. None of the other 44 possible treatment selections differed significantly between the pretests and posttests.

\section{User Evaluations}

Users evaluated the relative merits and value of the consultation reports with a 10 -item questionnaire using a 7-point Likert scale.

Both clinicians and experts found the suggestions to be extremely consistent with ethical guidelines, would recommend the reports to others, did not find the report to be redundant, and felt it quite unlikely that the suggestions would harm the client. They also thought that the report was adequately detailed, that it would enhance the effectiveness of relatively inexperienced clinicians, that it offered reasonable and relevant suggestions, and that it would help clinicians design a treatment protocol. However, the clinicians offered more positive ratings of the relevancy $[F(1,22)=5.39, p<.05]$ and helpfulness $[F(1,22)=9.08, p<.01]$ of the MR-E reports than did the experts.

\section{DISCUSSION}

This study supports MR-E's ability to provide advice that is substantially similar to that of the expert on whom the system was based. Its suggestions are in substantial 
agreement with those of the three human experts included in this study. Furthermore, its level of agreement with the three human experts was within the range of agreement found among those experts. At least in the two test cases, MR-E gave users expert advice. The next step would be to examine agreement with other cases and in real world settings, and these initial results recommend such an effort.

The impact of MR-E's expert advice on clinicians is significant. In terms of the functional hypotheses, clinicians significantly increased their agreement with MR-E after reading the consultation report. Changes in sensitivity and specificity elucidate the underlying factors in this improvement. Regarding specificity, clinicians were significantly more effective at correctly identifying existing functional hypotheses on posttest, though the $3 \%$ gain appears quite small. Clinician sensitivity-the ability to correctly reject nonrelevant functional hypothesesgrew by a clinically and statistically significant $24 \%$, however. Without MR-E, clinicians correctly rejected only 67 out of 100 nonrelevant functional hypotheses. With its assistance, they correctly rejected 91 out of 100 . In practical terms, the clinicians' increased sensitivity means that in their treatments they should tend to avoid nonrelevant, potentially unproductive options and to concentrate their efforts on the remaining relevant hypotheses.

MR-E also has a positive impact on preservice professionals who are learning about treatment in mental retardation. In a previous study (Hile \& Campbell, 1993), a significant increase in agreement on posttest was observed for preservice students, who agreed with MR-E at a level equivalent to that obtained during the pretest by the practicing clinicians in this study.

As well as affecting the choice of functional hypotheses, MR-E's report significantly influenced treatment selection by clinicians. Each of the four recommended treatments was endorsed more frequently after clinicians had read the report. It is not possible, with this data, to determine what impact MR-E would have on treatment implementation, but it appears likely that clinicians would follow MR-E's suggestions.

In addition to benefiting users, useful automated systems must also be accepted by those individuals. The evaluations obtained from the clinicians and experts alike suggest that MR-E's reports are acceptable. We noted that the clinicians offered more positive ratings than the experts on the report's relevance to the case and ability to help clinicians design a treatment protocol. Although the practical significance of this finding is not clear, it suggests that clinicians would find MR-E more helpful than experts would predict.

It is important to remember that this is an analogue study. The results provide strong initial support for MR-E's validity and lead us to recommend further studies on the system's clinical impact. In these additional studies, which will encompass a broader range of real cases from various clinical settings, we will analyze the impact of the full performance support system on clinician behavior.

Our results also emphasize the potential benefits of a PSS. With society's increasing interest in cost-effective treatments, such supports may help inexperienced clinicians function like their more experienced counterparts. By redirecting attention from nonrelevant functional hypotheses, the supports could increase the amount of time and effort clinicians can direct toward relevant hypotheses. And by providing support in a convenient and readily accessible form, they can quickly help clinicians develop expertise in the most current treatment techniques. Most importantly, by improving treatment planning, performance support systems such as MR-E can help clients lead more normal and productive lives.

\section{REFERENCES}

AXELROD, S. (1987). Functional and structural analyses of behavior: Approaches leading to reduced use of punishment procedures. $R e$ search in Developmental Disabilities, 8, 165-178.

CARR, C. (1991). Performance support systems: A new horizon for expert systems. AI Expert, 7(5), 44-49.

Fischer, P., Parides, M., \& Davies, M. (1993, Spring). Sensitivity and specificity. AACAP Research Notes in Child \& Adolescent Psychiatry, pp. 18-19.

GERY, G. J. (1991). Electronic performance support systems. Boston, MA: Weingarten.

Hile, M. G., \& Camprell, D. M. (1993, June). The impact of automated expert assistance on the training of mental retardation professionals. Paper presented at the meeting of the American Association on Mental Retardation, Washington, DC.

Hile, M. G., Campbell, D. M., Ghobary, B. B., \& Desrochers, M. N. (1993). Development of knowledge bases and the reliability of decision support for behavior treatment consultation for persons with mental retardation: The Mental Retardation-Expert. Behavior Research Methods, Instruments, \& Computers, 25, 195-198.

Hile, M. G., \& Desrochers, M. N. (in press). Decision support in designing behavioral treatments: The Mental Retardation-Expert. Computers in Human Behavior.

KazDIN, A. (1982). Single-case research design: Methods for clinical and applied settings. New York: Oxford University Press.

NoRUSIS, M. J. (1992). SPSS for Windows base system user's guide, Release 5.0. Chicago: SPSS. 